Article

\title{
Reduced Cytokine Release in Ex Vivo Response to Cilengitide and Cetuximab Is a Marker for Improved Survival of Head and Neck Cancer Patients
}

\author{
Susan Cedra, Susanne Wiegand, Marlen Kolb, Andreas Dietz and Gunnar Wichmann * \\ Department of Otolaryngology, Head and Neck Surgery, University of Leipzig, 04103 Leipzig, Germany; \\ scedra@gmx.net (S.C.); Susanne.Wiegand@medizin.uni-leipzig.de (S.W.); \\ Marlen.Kolb@medizin.uni-leipzig.de (M.K.); andreas.dietz@medizin.uni-leipzig.de (A.D.) \\ * Correspondence: gunnar.wichmann@medizin.uni-leipzig.de; Tel.: +49-341-972-1926 \\ Academic Editor: Helen M. Sheldrake
}

Received: 30 June 2017; Accepted: 2 September 2017; Published: 5 September 2017

\begin{abstract}
Targeting of $\alpha_{V} \beta 3$ and $\alpha_{V} \beta 5$ integrins by cilengitide may reduce growth of solid tumors including head and neck squamous cell carcinoma (HNSCC). Preclinical investigations suggest increased activity of cilengitide in combination with other treatment modalities. The only published trial in HNSCC (ADVANTAGE) investigated cisplatin, 5-fluorouracil, and cetuximab (PFE) without or with once (PFE+CIL1W) or twice weekly cilengitide (PFE+CIL2W) in recurrent/metastatic HNSCC. ADVANTAGE showed good tolerability of the cilengitide arms and even lower adverse events (AEs) compared to PFE but not the benefit in overall survival expected based on preclinical data. As we found in the FLAVINO assay, a short-time ex vivo assay for prediction of chemosensitivity, only a subgroup of HNSCC had an increased suppressive effect of cilengitide containing combination therapies on colony formation of epithelial cells $\left(\mathrm{CF}_{\mathrm{ec}}\right)$ and release of pro-angiogenetic and pro-inflammatory cytokines, whereas other HNSCC failed to respond. Response to $\alpha_{V} \beta 3$ and $\alpha_{V} \beta 5$ integrin targeting by cilengitide classifies HNSCC regarding outcome. We present FLAVINO data arguing for further development of cilengitide plus cetuximab in treatment of a subgroup of HNSCC potentially identified by the FLAVINO assay using a set of biomarkers for response evaluation.
\end{abstract}

Keywords: head and neck cancer; head and neck squamous cell carcinoma (HNSCC); predictive assay; chemoresponse ex vivo; cilengitide; integrin; $\alpha_{V} \beta 3$; targeted therapy; biomarker; interleukin 6; monocyte chemotactic protein-1

\section{Introduction}

As reviewed by Ahmedah and colleagues earlier this year, $\alpha_{V}$ integrins play a crucial role in the development, progression, and metastatic spread of head and neck squamous cell carcinoma (HNSCC), thus supporting the therapeutic potential of integrin targeting [1].

In our translational study, we focused on the efficacy of cilengitide applied either alone or as part of multi-component chemotherapy in HNSCC ex vivo. Here we present our findings including the involvement of pro-inflammatory and pro-angiogenic cytokines as potential prognostic markers.

Cilengitide, an N-methylated cyclic pentapeptide (cyclo-Arg-Gly-Asp-D-Phe-(N-methyl)-Val; EMD 121974; Merck KGaA, Darmstadt, Germany) containing the characteristic RGD-recognition sequence, specifically inhibits integrins $\alpha_{V} \beta 3$ and $\alpha_{V} \beta 5$ [2-4]. In the ex vivo short-time chemoresponse assay FLAVINO, we used alterations in colony formation and cytokine release of interleukin 6 (IL-6) and monocyte chemotactic protein-1 (MCP-1) as read-out for response of HNSCC to cilengitide plus cetuximab. 
IL-6 is a pleiotropic pro-inflammatory cytokine involved in numerous biological processes such as cell differentiation, wound healing, and apoptosis [5]. Dysregulated or excessive expressed IL-6 is associated with a variety of chronic inflammatory diseases and the development and maintenance of malignant tumors including HNSCC [6]. Elevated IL-6 levels in patients with HNSCC correlate with higher tumor stage, lymph node metastasis, increased proliferative tumor-activity, decreased immunologic response, and distinctive cachexia [7]. Furthermore, IL-6 supports vascular endothelial growth factor A production, resulting in enhanced tumor-angiogenesis [8]. There is evidence that IL-6 concentration decreases during successful anti-tumoral therapy, suggesting predictive qualities of the course of IL-6 concentration alteration [7].

As shown in previous studies by our laboratory, cilengitide modulates effects of MCP-1 production in HNSCC ex vivo [9,10], thus indicating MCP-1 as a valid molecule for testing. MCP-1 is a pleiotropic chemotactic cytokine that is essential for the recruitment of monocytes, macrophages, and natural killer cells during tissue injury and inflammation [11]. It is known that high concentrations of this CC-chemokine have impact on tumor environment and that it is intertwined with tumor invasiveness, progress of disease, metastatic spread, and tumor-angiogenesis [12,13]. MCP-1 demonstrates both tumor-supporting and anti-tumor effects due to its diverse influences on different cell types, but it is still not clear how it decides to act either way-probably concentration dependency plays a leading role in this question [10]. Many studies have linked elevated MCP-1 serum levels in patients with the presence, development, or recurrence of solid tumors, including advanced HNSCC, with significant lower overall survival (OS) and tumor-specific survival (TSS), indicating MCP-1 as a prognostic biomarker for solid tumors including HNSCC and clinical outcome [14].

\section{Results}

In analyses including specimens of 39 HNSCC patients (Table 1) we found a tremendous heterogeneity in the release of the cytokines IL-6 and MCP-1 after short-time culturing (72 h; see Material and Methods Section) of HNSCC.

Table 1. Characteristics of 39 histopathologically confirmed head and neck squamous cell carcinoma (HNSCC) of 34 male and 5 female patients (mean age at 60.3 years).

\begin{tabular}{cccccc}
\hline & $\mathbf{n}$ & $\mathbf{( \% )}$ & $\mathbf{n}$ & $\mathbf{( \% )}$ \\
\hline Localization & \multicolumn{5}{c}{ Tumor Stage } \\
\hline $\begin{array}{c}\text { hypopharynx/larynx } \\
\text { oropharynx }\end{array}$ & 12 & $(30.8 \%)$ & UICC I & 2 & $(5.1 \%)$ \\
nasopharynx & 24 & $(61.5 \%)$ & UICC II & 6 & $(15.4 \%)$ \\
oral cavity & 1 & $(2.6 \%)$ & UICC III & 7 & $(17.9 \%)$ \\
\hline T Category & 2 & $(5.1 \%)$ & UICC IV & 24 & $(61.5 \%)$ \\
\hline T1 & 5 & $(12.8 \%)$ & Lifetime Tobacco Exposure (Pack Years, py) \\
T2 & 7 & $(17.9 \%)$ & $1-20$ py & 8 & $(20.5 \%)$ \\
T3 & 14 & $(35.9 \%)$ & $21-40$ py & 14 & $(7.7 \%)$ \\
T4a & 13 & $(33.3 \%)$ & $41-60$ py & 8 & $(20.5 \%)$ \\
T4b & 0 & $(0.0 \%)$ & $>60$ py & 4 & $(10.3 \%)$ \\
& & \multicolumn{5}{c}{ no information } & 2 & $(5.1 \%)$ \\
\hline N Category & & Alcohol Consumption (g/day) \\
\hline N0 & 16 & $(41.0 \%)$ & 0 & 4 & $(10.3 \%)$ \\
N1 & 3 & $(7.7 \%)$ & $<30$ & 14 & $(35.9 \%)$ \\
N2a & 1 & $(2.6 \%)$ & $31-60$ & 8 & $(20.5 \%)$ \\
N2b & 6 & $(15.4 \%)$ & $\geq 60$ & 11 & $(28.2 \%)$ \\
N2c & 12 & $(30.8 \%)$ & no information & 2 & $(5.1 \%)$ \\
N3 & 1 & $(2.6 \%)$ & & \\
\hline
\end{tabular}


This is consistent with earlier findings in investigations applying cilengitide either alone or in combination with other treatments to solid tumors in vitro and in vivo [15-23]. We also detected varying efficacy regarding reduction of colony formation. The heterogeneity in response of HNSCC to cilengitide and in particular to cilengitide in binary combination with cetuximab (Cil+E) is substantial (Figure 1).

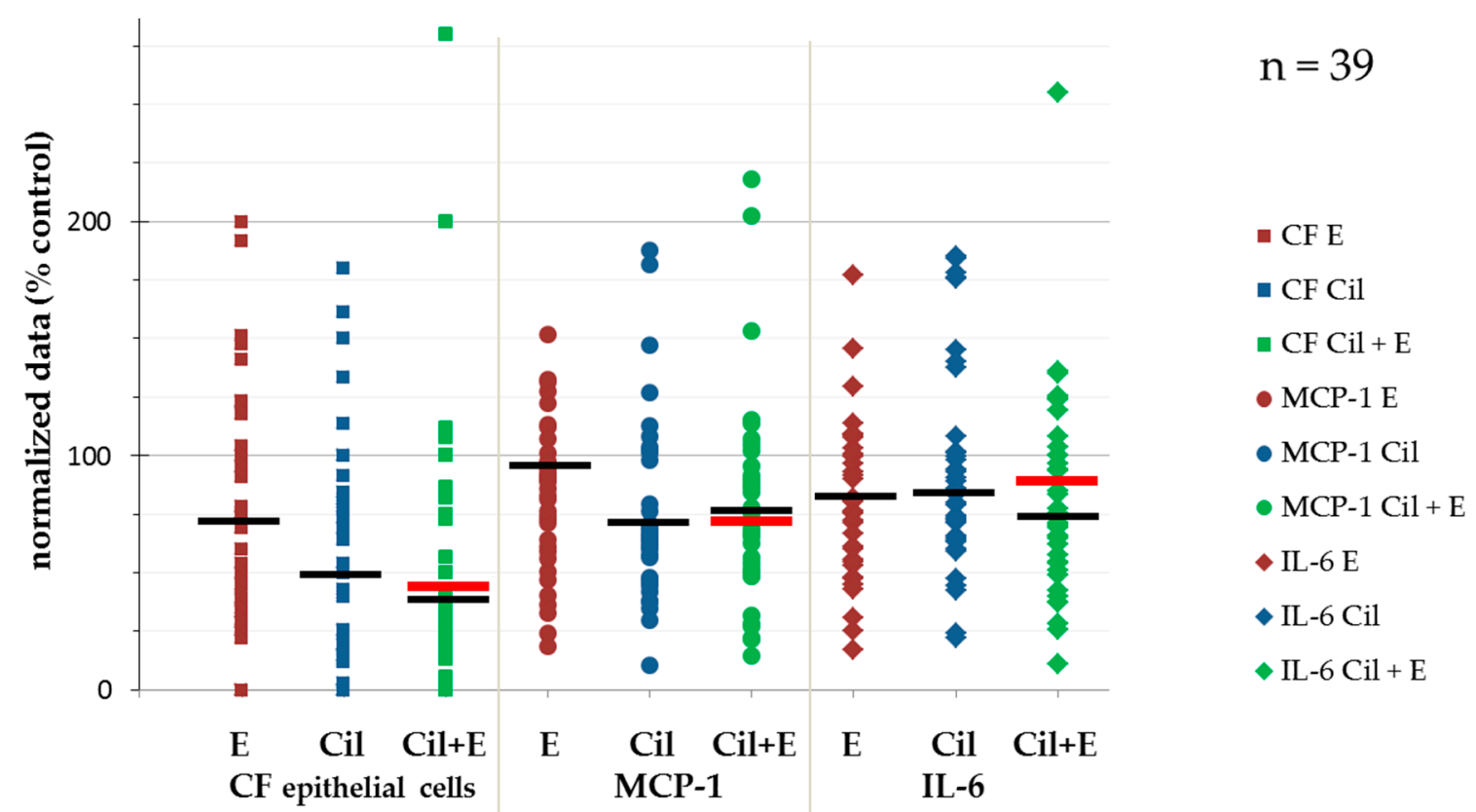

Figure 1. Heterogeneity in response of $39 \mathrm{HNSCC}$ in the FLAVINO assay according to the readouts of colony formation (CF), release of MCP-1, and interleukin 6. Black lines represent median values, red lines represent cutoff values identified in receiver operator characteristic (ROC) analyses (Figures S1-S3). Abbreviations: CF, colony formation; IL-6, interleukin 6; MCP-1, monocyte chemotactic protein-1; Cil, cilengitide; E, Erbitux (cetuximab); Cil+E, cilengitide combined with Erbitux (cetuximab).

We analyzed the outcome of patients under study regarding OS using receiver operator characteristic (ROC) curves (Supplementary Figures S1-S3) and found significant areas under the curve (AUC) regarding predicted survival for MCP- $1 \leq 75 \%$, and statistical trends for CF $\leq 45 \%$, and IL- $6 \leq 90 \%$ of controls. The grouping of HNSCC patients according to these cutoff values revealed significantly different TSS and OS of patients (Figure 2). Suppression of MCP-1 concentration below $75 \%$ of controls and IL- 6 concentration below $90 \%$ of controls under treatment of cetuximab plus cilengitide were associated with significantly improved OS (MCP-1 $p<0.006$; IL-6 $p<0.007$ ) and TSS (MCP-1 $p<0.08$; IL-6 $p<0.004$ ). This trend for improved survival was also seen in patients whose HNSCC responded to Cil+E with suppression of $\mathrm{CF}_{\mathrm{ec}}$ below $45 \%$ of controls $(\mathrm{OS} p<0.066$; TSS $p<0.061$ ). Since readouts of cytokine release in triplicate measurements achieved more consistent and objective results than manual microscopic cell counting for $\mathrm{CF}_{\mathrm{ec}}$ [9], measurement of IL-6 and MCP-1 might be a suitable new strategy for the prediction of patients' likely outcomes. Independent from the chosen readout, the statistical analyses using Fisher's exact test revealed no significant difference in distribution of values above and below the cutoff regarding response of HNSCC samples taken from early stages (UICC I and II) vs. locoregional advanced HNSCC stages (UICC III and IV) or T categories T1 and T2 vs. T3 and T4 to either cilengitide, cetuximab, or Cil+E and the detected prognostic value (all $p>0.387$ ). Only slight and insignificant differences in the patients' OS associated with UICC stage and T category were found in Kaplan Meier curves comparing binary classified patients according to the cutoff for the readouts CFec $(p=0.951$ and $p=0.465)$, or the release of IL-6 $(p=0.955$ and $p=0.501)$ or MCP-1 $(p=0.883$ and $p=0.771)$. 

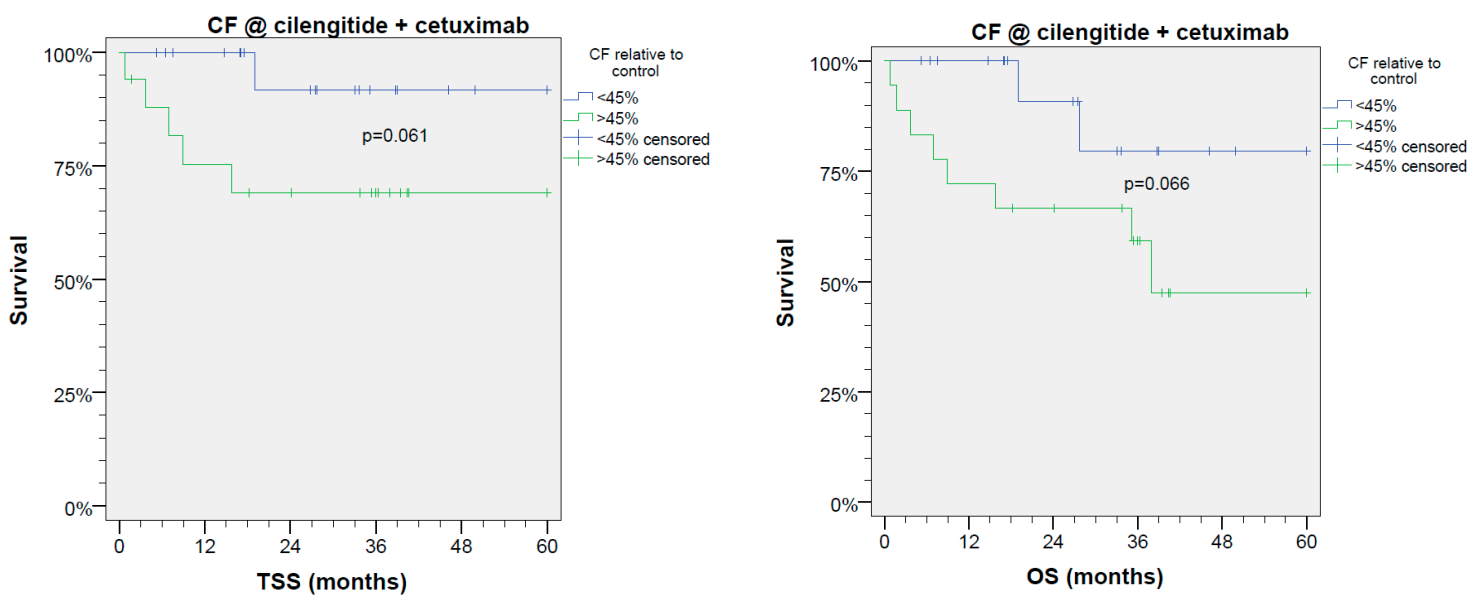

(a)

(b)

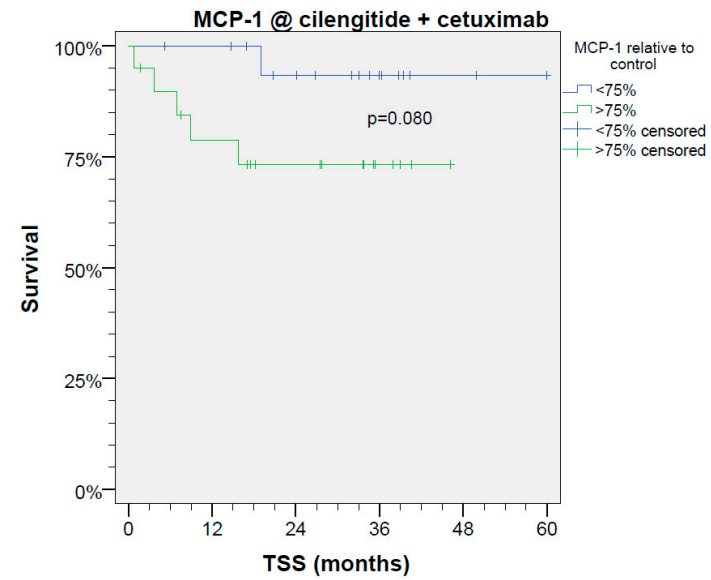

(c)

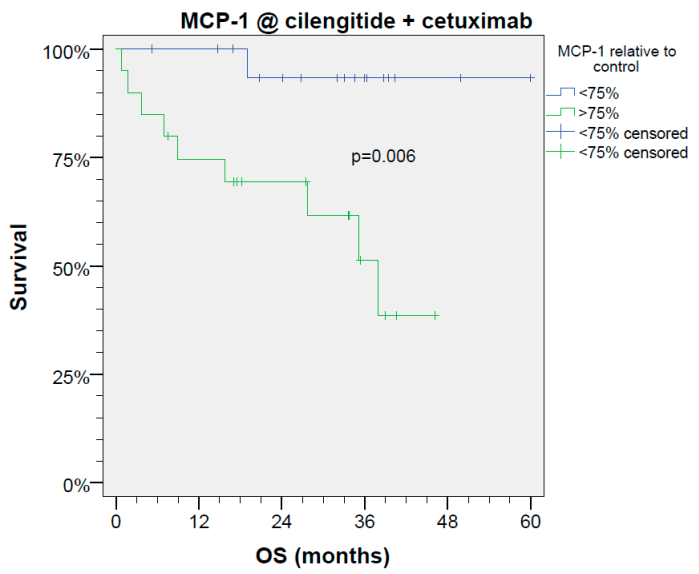

(d)

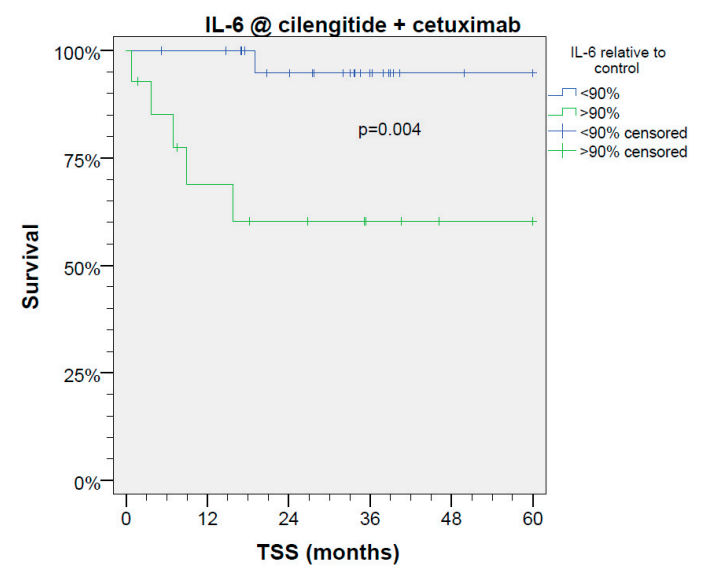

(e)

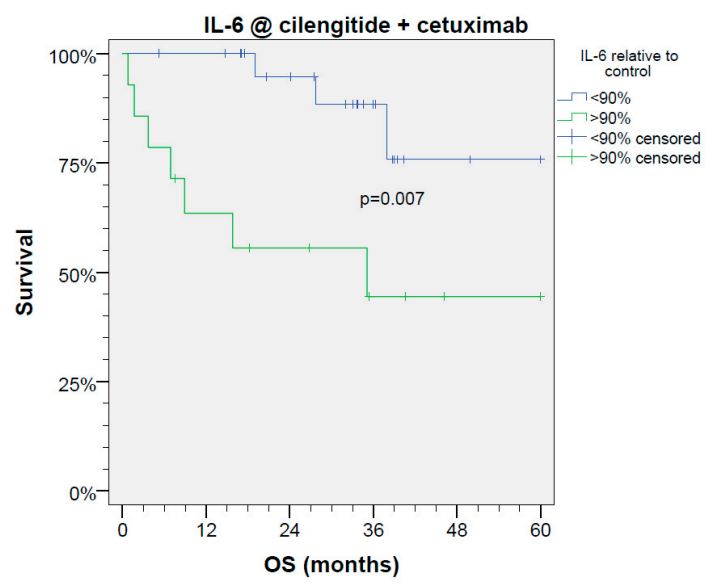

(f)

Figure 2. Colony formation of epithelial cells ( $\mathrm{CFec}$ ) and release of MCP-1 and IL-6 are prognostic factors for tumor-specific $(\mathbf{a}, \mathbf{c}, \mathbf{e})$ and overall survival $(\mathbf{b}, \mathbf{d}, \mathbf{f})$ of HNSCC patients. $(\mathbf{a}, \mathbf{b})$ Colony formation; $(\mathbf{c}, \mathbf{d})$ release of MCP-1, (e,f) release of IL-6 as optimized classifiers for survival derived from significant receiver-operating characteristic curves obtained from $n=39$ HNSCC patients.

\section{Discussion}

Our set of data demonstrates a huge heterogeneity of head and neck cancer cells in response to Cil+E. A strongly reduced release of IL- 6 and MCP-1 by Cil+E treated HNSCC is demonstrated 
as being a potential classifier for the patients' OS and TSS. Our results also imply the existence of a subgroup of patients potentially benefitting from combined Cil+E treatment.

A more detailed look at results obtained in ADVANTAGE $[24,25]$ reveals a $10 \%$ higher objective response rate (ORR) in PFE + CIL1W vs. PFE of $42 \%$ (95\% confidence interval, CI, 30-55\%) vs. 32\% (95\% CI 21-45\%) corresponding to an odds ratio (OR) of 1.516 (95\% CI 0.732-3.141) in the independent read (sensitivity analysis). This higher ORR in PFE+CIL1W vs. PFE was accompanied by a prolonged median time to treatment failure (5.6 vs. 4.2 months) but led to a slightly increased hazard ratio (HR) for progression-free survival (PFS; HR 1.15, 95\% CI 0.74-1.79; $p=0.528$ ), whereas the OS of patients treated with PFE+CIL1W was not inferior (HR 0.94, 95\% CI 0.61-1.47; $p=0.800$ ).

As the primary objective of the trial (i.e., reasonably improved OS) was not met, the investigators concluded that further development of PFE+CIL1W or PFE+CIL2W in R/M HNSCC cannot be recommended. Does this mean that also further development of the binary combination of cilengitide plus cetuximab for treatment of R/M HNSCC should not be recommended?

Our findings in the FLAVINO study [9] and the here shown suitability of these data to classify HNSCC regarding their prognosis together with the data published for ADVANTAGE strongly suggest considerable therapeutic potential of cilengitide in combination with cetuximab in preselected HNSCC patients potentially identified using the outcome measured in the FLAVINO assay. As cilengitide even in combination with multiple other chemotherapeutics was well tolerated $[21,24]$, there might be a place for cilengitide in binary combination with cetuximab (Cil+E) instead of cetuximab monotherapy in R/M HNSCC without adequate general health to receive PFE or after failure of platinum-based regimens. Future research may reveal if cilengitide deserves another chance in R/M HNSCC.

\section{Materials and Methods}

\subsection{Patient Characteristics}

HNSCC samples of 43 patients were included in this study. With patients' informed consent, biopsies of tumor tissue were obtained during surgery or panendoscopy. All patients received therapy according to the consented decision made by the Head and Neck Cancer Tumor Board of the University Hospital Leipzig according to German therapy guidelines; none of the patients were treated with cilengitide and/or cetuximab. Thirty-nine histopathologically confirmed HNSCC of these 43 patients, 34 male and 5 female patients (mean age of 60.3 years; Table 1), could be analyzed regarding cytokine production and colony formation after treating the specimens with either Cil or $\mathrm{E}$ alone or combined (Cil+E).

\subsection{FLAVINO-Assay}

The same procedures as the protocol of the FLAVINO assay were used, as previously described [9]. Freshly obtained tumor specimens were put into phenol red- and riboflavin-free medium supplemented with $10 \%$ fetal calf serum and antibiotics (TM). After mechanical disintegration and digestion by $230 \mathrm{mU} / \mathrm{mL}$ collagenase IV (Sigma-Aldrich, Deisenhofen, Germany), 10,000 viable HNSCC cells were added to triplicate wells coated with human laminin (Roche, Germany) containing either $66.7 \mu \mathrm{g} / \mathrm{mL}$ E, $10 \mu \mathrm{M}$ Cil, Cil+E, or (for reference) TM alone, adjusting the total volume to $300 \mu \mathrm{L}$. Supernatants harvested after 3 days were analyzed by ELISA and adherent cells ethanol-fixated and underwent pan-cytokeratin staining by FITC-labeled antibodies and counting of green fluorescent colonies of epithelial cells. Thirty-nine HNSCC had adherent growth (mean $\mathrm{CF}_{\mathrm{ec}} \geq 4$ /well in triplicate controls).

\subsection{ELISA}

The cytokines IL-6 and MCP-1 released into supernatants were measured using indirect sandwich ELISAs (OptEIA Kits; BD Biosciences, Heidelberg, Germany) following the manufacturer's instructions and using tetra-methyl benzidine as substrate. The optical density of each well was determined 
measuring the optical density at $\lambda_{1}=450 \mathrm{~nm}$ and $\lambda_{2}=620 \mathrm{~nm}$ on the Synergy $2^{\mathrm{TM}}$ multi-mode microplate reader (BioTek Instruments, Inc., Winooski, VT, USA) according to 4-parameter calibration curves calculated using Gen5 software (BioTek). The lower limit of detection (LLD) was $\leq 4 \mathrm{pg} / \mathrm{mL}$, the lower limit of quantification (LLQ) was $\leq 7 \mathrm{pg} / \mathrm{mL}$ for both cytokines, two orders of magnitude below median cytokine concentrations in untreated controls [9].

\subsection{Statistical Analysis}

All data shown are based on triplicate measurements. Differences were compared by Student's $t$-test for paired samples. The maximum Youden scores (sensitivity plus specificity) were used for finding in ROC curves with significant areas under the curve the optimum cutoff for binary classification of HNSCC patients for survival data analyses according to the Kaplan-Meier method by applying the log-rank test. Statistics were done using SPSS Statistics for Windows, version 20.0.0 (SPSS Inc., Chicago, IL, USA). $P \leq 0.05$ was regarded as significant.

\subsection{Ethical Approval}

The study was approved by the Ethics Committee of the Medical Faculty of the University Leipzig (study codes 180-2007, 201-10-12072010, and 341-15-05102015) and performed in accordance with the ethical standards as laid down in the 1964 declaration of Helsinki and its later amendments.

\section{Conclusions}

In a subgroup of HNSCC, the ex vivo targeting $\alpha_{V} \beta 3$ and $\alpha_{V} \beta 5$ integrins by cilengitide and EGFR by cetuximab leads to reduced $\mathrm{CF}_{\mathrm{ec}}$ and release of IL- 6 and MCP-1, thus proving that cilengitide in binary combination with cetuximab does have potential in the treatment of this subgroup. As response in the FLAVINO assay was associated with response in vivo $[9,26]$, the FLAVINO assay may allow for the detection of HNSCC patients that could benefit from the combined targeted therapy. In this respect, IL-6 and MCP-1 gain significance as prognostic biomarkers for improved overall survival when cetuximab plus cilengitide achieve a decrease of IL- 6 production $<90 \%$ and of MCP-1 production $<75 \%$ relative to control.

Future research may focus on the development of cilengitide plus cetuximab in preselected R/M HNSCC.

Supplementary Materials: The following are available online at http:/ /www.mdpi.com/2072-6694/9/9/117/s1. Supplementary Figures S1-S3.

Acknowledgments: The study was supported by Merck Serono GmbH. The authors received funds for covering the costs to publish in open access from the Medical Faculty of the University of Leipzig. We thank all patients and their families for their participation in this study and Anett Reiche for technical assistance.

Author Contributions: Gunnar Wichmann conceived and designed the experiments; Susan Cedra and Gunnar Wichmann performed the experiments; Susan Cedra and Gunnar Wichmann analyzed the data; Susan Cedra, Marlen Kolb, Susanne Wiegand, Andreas Dietz, and Gunnar Wichmann wrote the paper.

Conflicts of Interest: GW received funding for the project "Cilengitide in HNSCC ex vivo" and honoraria and travel expenses from Merck Serono GmbH. The other authors all declare no conflict of interest. The founding sponsor Merck has no role in the design of the study; in the collection, analyses, or interpretation of data; in the writing of the manuscript, or in the decision to publish the results.

\section{References}

1. Ahmedah, H.T.; Patterson, L.H.; Shnyder, S.D.; Sheldrake, H.M. RGD-binding integrins in head and neck cancers. Cancers 2017, 9, 56. [CrossRef] [PubMed]

2. Mas-Moruno, C.; Rechenmacher, F.; Kessler, H. Cilengitide: The first anti-angiogenic small molecule drug candidate design, synthesis and clinical evaluation. Anti-Cancer Agents Med. Chem. 2010, 10, 753-768. [CrossRef] 
3. Goodman, S.L.; Holzemann, G.; Sulyok, G.A.G.; Kessler, H. Nanomolar small molecule inhibitors for alphav(beta)6, alphav(beta)5, and alphav(beta)3 integrins. J. Med. Chem. 2002, 45, 1045-1051. [CrossRef] [PubMed]

4. Dechantsreiter, M.A.; Planker, E.; Matha, B.; Lohof, E.; Holzemann, G.; Jonczyk, A.; Goodman, S.L.; Kessler, H. N-Methylated cyclic RGD peptides as highly active and selective alpha(V)beta(3) integrin antagonists. J. Med. Chem. 1999, 42, 3033-3040. [CrossRef] [PubMed]

5. Tanaka, T.; Kishimoto, T. The biology and medical implications of interleukin-6. Cancer Immunol. Res. 2014, 2, 288-294. [CrossRef] [PubMed]

6. Lippitz, B.E. Cytokine patterns in patients with cancer: A systematic review. Lancet Oncol. 2013, 14, e218-e228. [CrossRef]

7. Riedel, F.; Zaiss, I.; Herzog, D.; Gotte, K.; Naim, R.; Hormann, K. Serum levels of interleukin-6 in patients with primary head and neck squamous cell carcinoma. Anticancer Res. 2005, 25, 2761-2765. [PubMed]

8. Nagasaki, T.; Hara, M.; Nakanishi, H.; Takahashi, H.; Sato, M.; Takeyama, H. Interleukin-6 released by colon cancer-associated fibroblasts is critical for tumour angiogenesis: Anti-interleukin- 6 receptor antibody suppressed angiogenesis and inhibited tumour-stroma interaction. Br. J. Cancer 2014, 110, 469-478. [CrossRef] [PubMed]

9. Wichmann, G.; Cedra, S.; Schlegel, D.; Kolb, M.; Wiegand, S.; Boehm, A.; Hofer, M.; Dietz, A. Cilengitide and cetuximab reduce cytokine production and colony formation of head and neck squamous cell carcinoma cells ex vivo. Anticancer Res. 2017, 37, 521-527. [CrossRef] [PubMed]

10. Wichmann, G.; Korner, C.; Boehm, A.; Mozet, C.; Dietz, A. Stimulation by monocyte chemoattractant protein-1 modulates the ex vivo colony formation by head and neck squamous cell carcinoma cells. Anticancer Res. 2015, 35, 3917-3924. [PubMed]

11. Deshmane, S.L.; Kremlev, S.; Amini, S.; Sawaya, B.E. Monocyte chemoattractant protein-1 (MCP-1): An overview. J. Interferon Cytokine Res. 2009, 29, 313-326. [CrossRef] [PubMed]

12. Ji, W.-T.; Chen, H.-R.; Lin, C.-H.; Lee, J.-W.; Lee, C.-C. Monocyte chemotactic protein 1 (MCP-1) modulates pro-survival signaling to promote progression of head and neck squamous cell carcinoma. PLoS ONE 2014, 9, e88952. [CrossRef] [PubMed]

13. Salcedo, R.; Ponce, M.L.; Young, H.A.; Wasserman, K.; Ward, J.M.; Kleinman, H.K.; Oppenheim, J.J.; Murphy, W.J. Human endothelial cells express CCR2 and respond to MCP-1: Direct role of MCP-1 in angiogenesis and tumor progression. Blood 2000, 96, 34-40. [PubMed]

14. Wang, H.; Zhang, Q.; Kong, H.; Zeng, Y.; Hao, M.; Yu, T.; Peng, J.; Xu, Z.; Chen, J.; Shi, H. Monocyte chemotactic protein-1 expression as a prognosic biomarker in patients with solid tumor: A meta-analysis. Int. J. Clin. Expe. Pathol. 2014, 7, 3876-3886.

15. Abdollahi, A.; Griggs, D.W.; Zieher, H.; Roth, A.; Lipson, K.E.; Saffrich, R.; Grone, H.-J.; Hallahan, D.E.; Reisfeld, R.A.; Debus, J.; et al. Inhibition of alpha(v)beta3 integrin survival signaling enhances antiangiogenic and antitumor effects of radiotherapy. Clin. Cancer Res. 2005, 11, 6270-6279. [CrossRef] [PubMed]

16. Buerkle, M.A.; Pahernik, S.A.; Sutter, A.; Jonczyk, A.; Messmer, K.; Dellian, M. Inhibition of the alpha-nu integrins with a cyclic RGD peptide impairs angiogenesis, growth and metastasis of solid tumours in vivo. Br. J. Cancer 2002, 86, 788-795. [CrossRef] [PubMed]

17. Burke, P.A.; DeNardo, S.J.; Miers, L.A.; Lamborn, K.R.; Matzku, S.; DeNardo, G.L. Cilengitide targeting of alpha(v)beta(3) integrin receptor synergizes with radioimmunotherapy to increase efficacy and apoptosis in breast cancer xenografts. Cancer Res. 2002, 62, 4263-4272. [PubMed]

18. MacDonald, T.J.; Taga, T.; Shimada, H.; Tabrizi, P.; Zlokovic, B.V.; Cheresh, D.A.; Laug, W.E. Preferential susceptibility of brain tumors to the antiangiogenic effects of an alpha(v) integrin antagonist. Neurosurgery 2001, 48, 151-157. [PubMed]

19. Mitjans, F.; Meyer, T.; Fittschen, C.; Goodman, S.; Jonczyk, A.; Marshall, J.F.; Reyes, G.; Piulats, J. In vivo therapy of malignant melanoma by means of antagonists: V integrins. Int. J. Cancer 2000, 87, 716-723. [CrossRef]

20. Raguse, J.-D.; Gath, H.J.; Bier, J.; Riess, H.; Oettle, H. Cilengitide (EMD 121974) arrests the growth of a heavily pretreated highly vascularised head and neck tumour. Oral Oncol. 2004, 40, 228-230. [CrossRef] [PubMed] 
21. Stupp, R.; Hegi, M.E.; Gorlia, T.; Erridge, S.C.; Perry, J.; Hong, Y.-K.; Aldape, K.D.; Lhermitte, B.; Pietsch, T.; Grujicic, D.; et al. Cilengitide combined with standard treatment for patients with newly diagnosed glioblastoma with methylated MGMT promoter (CENTRIC EORTC 26071-22072 study): A multicentre, randomised, open-label, phase 3 trial. Lancet Oncol. 2014, 15, 1100-1108. [CrossRef]

22. Wang, J.T.; Liu, Y.; Kan, X.; Liu, M.; Lu, J.G. Cilengitide, a small molecule antagonist, targeted to integrin alphanu inhibits proliferation and induces apoptosis of laryngeal cancer cells in vitro. Eur. Arch. Otorrinolaringol. 2014, 271, 2233-2240. [CrossRef] [PubMed]

23. Heiduschka, G.; Lill, C.; Schneider, S.; Seemann, R.; Kornek, G.; Schmid, R.; Kotowski, U.; Thurnher, D. The effect of cilengitide in combination with irradiation and chemotherapy in head and neck squamous cell carcinoma cell lines. Strahlenther. Onkol. 2014, 190, 472-479. [CrossRef] [PubMed]

24. Vermorken, J.B.; Guigay, J.; Mesia, R.; Trigo, J.M.; Keilholz, U.; Kerber, A.; Bethe, U.; Picard, M.; Brummendorf, T.H. Phase I/II trial of cilengitide with cetuximab, cisplatin and 5-fluorouracil in recurrent and/or metastatic squamous cell cancer of the head and neck: Findings of the phase I part. Br. J. Cancer 2011, 104, 1691-1696. [CrossRef] [PubMed]

25. Vermorken, J.B.; Peyrade, F.; Krauss, J.; Mesia, R.; Remenar, E.; Gauler, T.C.; Keilholz, U.; Delord, J.P.; Schafhausen, P.; Erfan, J.; et al. Cisplatin, 5-fluorouracil, and cetuximab (PFE) with or without cilengitide in recurrent/metastatic squamous cell carcinoma of the head and neck: Results of the randomized phase I/II ADVANTAGE trial (phase II part). Ann. Oncol. 2014, 25, 682-688. [CrossRef] [PubMed]

26. Wichmann, G.; Dietz, A. Präklinische Modelle zur Etablierung innovativer Therapiestrategien: Ex vivo-Testung der Chemo und Immunresponse von Kopf-Hals-Tumoren. HNO 2016, 64, 460-469. [CrossRef] [PubMed]

(C) 2017 by the authors. Licensee MDPI, Basel, Switzerland. This article is an open access article distributed under the terms and conditions of the Creative Commons Attribution (CC BY) license (http:/ / creativecommons.org/licenses/by/4.0/). 